\title{
Brechas de género en la Educación Secundaria en población vulnerable de Chihuahua
}

\section{Gender gaps in middle schools in vulnerable population of Chihuahua}

\begin{abstract}
Romelia Hinojosa Luján Departamento de Investigación de la Secretaría de Educación, Cultura y Deporte del Gobierno del Estado de Chihuahua romehinojosa@yahoo.com.mx

Alicia Fonseca Venegas Departamento de Investigación de la Secretaría de Educación, Cultura y Deporte del Gobierno del Estado de Chihuahua aliciafonsecav@yahoo.com.mx

Óscar Cázares Delgado Departamento de Investigación de la Secretaría de Educación, Cultura y Deporte del Gobierno del Estado de Chihuahua oscar.cazares.d@gmail.com
\end{abstract}

\begin{abstract}
Resumen
Este trabajo aborda aspectos de la Calidad de Vida de estudiantes de Educación Secundaria en la región serrana del Estado de Chihuahua desde la perspectiva de género. Se buscó establecer las brechas de género existentes entre la información que reportan hombres y mujeres al cuestionárseles de aspectos específicos sobre salud y educación. Se seleccionó una muestra representativa aleatoria de estudiantes de 19 escuelas localizadas en 13 comunidades con población indígena a la que se les aplicó un cuestionario en el que se abarcan aspectos de salud, familia y educación. Los resultados apuntan a la existencia de brechas que tienen que ver con construcciones sociales de lo que tradicionalmente se adscribe a la masculinidad y a la feminidad.
\end{abstract}

Palabras clave

Diferencias de género, Educación Indígena, Educación Secundaria (Educación Media).

\begin{abstract}
This paper addresses aspects of the Quality of Life of Secondary Education students in the mountain region of the State of Chihuahua from a gender perspective. We sought to establish existing gender gaps amongst the information reported by men and women when questioned about specific aspects of health and education. A random representative sample of students from 19 schools located in 13 communities with an indigenous population was selected and a survey was applied, covering the topics of health, family, and education. The results point to the existence of gaps that have to do with social constructs traditionally ascribed to masculinity and femininity.
\end{abstract}




\section{Keywords}

Gender differences, Indigenous Education, Secondary Education (Middle school).

\section{Introducción}

Las políticas educativas que enmarcan a la educación secundaria mexicana destacan, en las últimas dos décadas, un fortalecimiento de ésta a través de la generalización y obligatoriedad de la misma. Los últimos pasos dados en el nivel, lo ubican como "un nivel estratégico para orientar el nuevo rumbo del sistema educativo nacional de acuerdo con las necesidades de la población que deberá atender y los requerimientos de calidad que deberá cubrir" (López \& Angulo, 2006, p. 1427).

Sin embargo, con todos los esfuerzos para expandir el servicio, en la actualidad se concentran problemáticas importantes como: la calidad, la pertinencia (Jarque, 2001; Tedesco \& López, 2002) y la permanencia principalmente (Schmelkes, 2012). Esto no es privativo de México pues a nivel internacional se siguen encontrando niveles alarmantes de deserción (Zorrilla Fierro, 2004). Es necesario precisar que el abandono y la deserción, así como la falta de calidad de las escuelas se concentra en los estratos de los y las estudiantes más pobres y vulnerables: mujeres y minorías étnicas (Román, 2009).
Si bien existen datos que pueden dar idea del comportamiento o caracterización de la juventud que cursa la educación secundaria en nuestra entidad y su comparación con las cifras nacionales (INEE, 2013), los estudios privilegian los promedios estatales en descuido de los números municipales. Chihuahua vive dos realidades alternas: una gran extensión territorial y número de municipios con un Índice de Desarrollo Humano "Alto" y "Muy alto", sin embargo, casi la mitad de los municipios que integran la entidad están comprendidos entre el grupo de aquéllos con Índice de Desarrollo Humano "Medio" y "Bajo". La gráfica 1 nos muestra un comparativo entre las situaciones de los estados de la República en 2010 y la situación que guarda nuestra entidad en la que destaca una brecha de desigualdad entre el municipio con mejor IDH: Chihuahua con .806 y el municipio con menor IDH Batopilas con un .387. Al comparar los municipios chihuahuenses se percibe una desigualdad que se objetiva en un 52\% (PNUD, 2014), pero al comparar la entidad con otros estados, se aprecia el enorme reto chihuahuense de superar la desigualdad. 


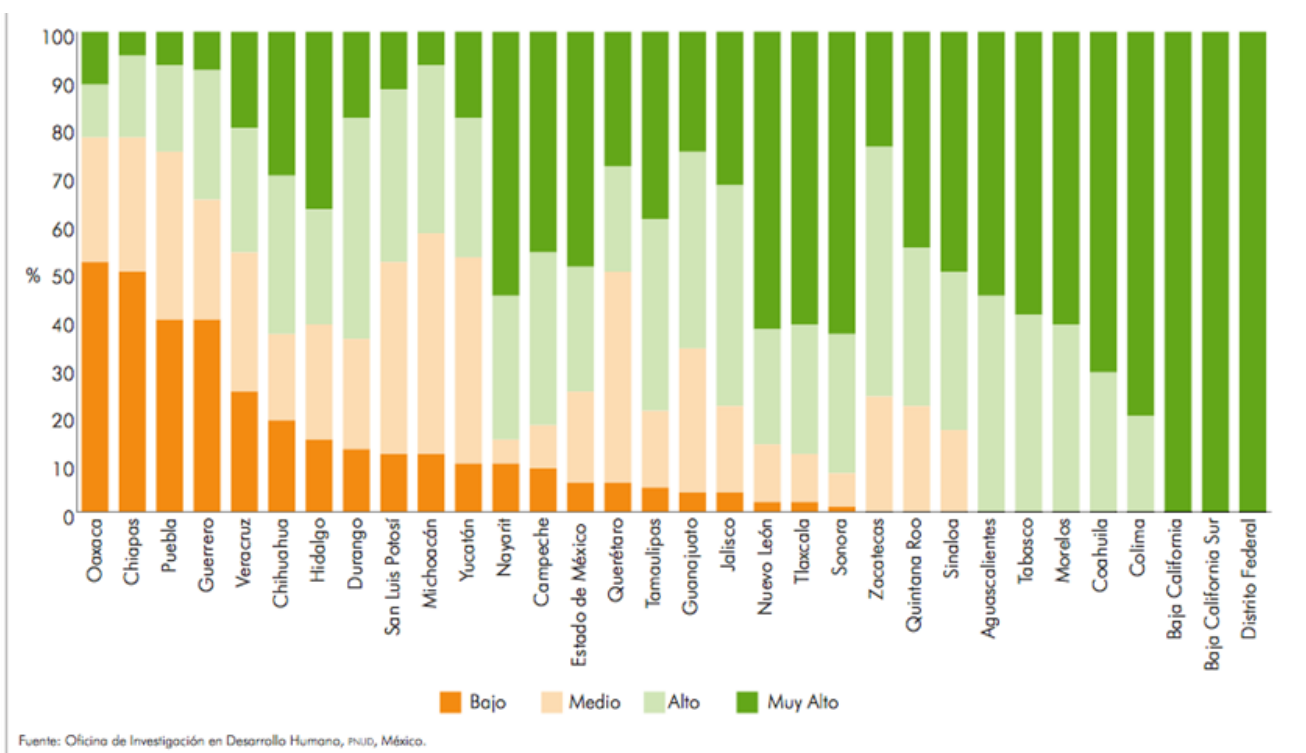

Gráfica 1. Porcentaje de municipios en cada entidad según categoría de desarrollo humano (2010)(PNUD, 2014b).

La localización geográfica de los municipios con menor IDH se registra en la Ilustración 1 que nos proporciona el PNUD y que de manera directa corresponde con aquellos municipios chihuahuenses que tienen habitantes indígenas (Ilustración 2): Guachochi con un 25.1 y Guadalupe y Calvo con una concentración de $12.9 \%$ de hablantes de lengua indígena. Se localizan un grupo de nueve municipios donde se concentra el $50 \%$ de la población hablante de lengua indígena del estado en total: Juárez, Urique, Balleza, Bocoyna, Chihuahua, Batopilas, Carichí, Guazapares y Morelos. En Chihuahua y Juárez ha sido por la migración indígena, en los otros municipios en asentamiento tradicionales (INEGI, 2004).

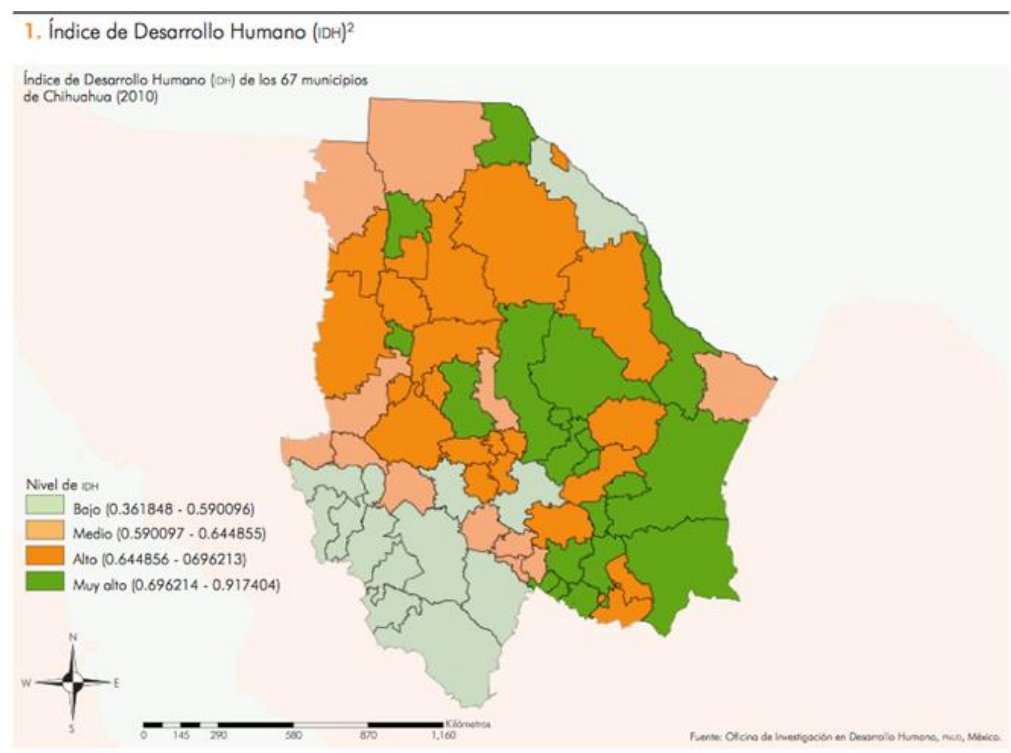

Ilustración 1 (PNUD, 2014). 


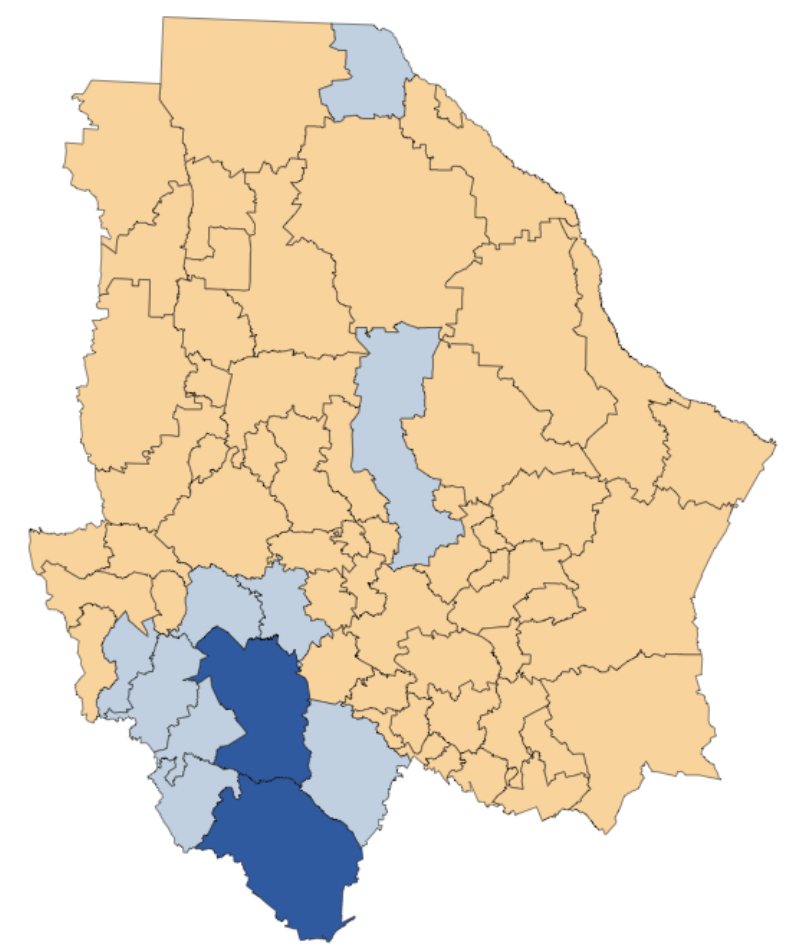

Ilustración 2 (INEGI, 2004).

Al tener como componente del Índice de Desarrollo Humano el aspecto educativo se puede establecer la importancia que ésta tiene para toda sociedad. En estos contextos de alta marginalidad es importante visualizar cifras de desigualdad que permitan establecer áreas de oportunidad. El nivel educativo de la población es un factor fundamental del nivel del desarrollo de un país.

Calidad de vida es un estado de satisfacción general derivado de la realización de las potencialidades de la persona. Posee aspectos subjetivos y aspectos objetivos. Es una sensación subjetiva de bienestar físico psicológico y social. Incluye como aspectos subjetivos la intimidad la expresión emocional la seguridad percibida la productividad personal y la salud objetiva. Como aspectos objetivos el bienestar material las relaciones armónicas con el ambiente físico y social y con la comunidad y la salud objetivamente percibida (Aroila, 2003, p. 163).

En el concepto Calidad de Vida, se incluyen, además de condicionantes subjetivos, otros objetivos como lo es la educación. También otros factores como la salud física, la salud mental, las condiciones educativas y un contexto sano. Por ello es que en este trabajo se hace énfasis a los indicadores medidos a través de la categoría Calidad de Vida como son salud, activación física, visión, etc. y las diferencias que se establecen con la variable género.

La focalización del estudio se dio hacia un grupo altamente vulnerable: la población indígena y rural del estado de Chihuahua. Se puede afirmar que los estudiantes que participaron en el estudio tienen alto grado de vulnerabilidad. La investigación en conjunto no contemplaba como indicador de análisis al género, sin embargo, la perspectiva de género brinda otra óptica de la problemática, por ello es que se asumió como posibilidad de análisis.

Uno de los factores importantes en el proceso de vulnerabilidad es el género. "La vulnerabilidad de género, por ejemplo, está anclada a un sistema de significación donde la condición social de mujeres y hombres debe 
responder a determinadas condiciones sociales que han sido impuestas y transformadas culturalmente; esta representación de roles definidos y estereotipados ha constituido una fuente de malestar (Burin et al.,) que provoca estados de indefensión y desesperanza frente a condiciones económicas y sociales que subyacen a la realidad de las personas" (Oswald-Spring et al., 2004, p. 279).

Significaciones que construyen las condiciones que hombres y mujeres deben de tener de acuerdo a lo que la sociedad establece como deseable. Construcciones culturales y sociales que se anclan en el sexo biológico y que generan imaginarios en torno a lo que una persona debe de ser únicamente por su fisiología. Marta Lamas expresa que la oposición binaria entre hombre/mujer instaura una simbolización en todos los aspectos de la vida llamada género. La sociedad finca sobre los cuerpos sexuados ideas de lo que debe ser "propio" para cada sexo (Lamas, 1995). La constitución de género origina desigualdad y discriminación en las relaciones sociales y problemáticas creadas a partir del cuerpo que se traducen en ideas y prejuicios. El hombre ocupa una posición hegemónica sobre la mujer (Lamas, 1996).

La desigualdad por este motivo produce brechas de género. Es decir, diferencias en las tasas masculina y femenina en la categoría de una variable (Género, s/d). Las brechas de género son construidas sobre las diferencias biológicas y son el producto histórico de actitudes y prácticas discriminatorias tanto individuales como sociales e institucionales, que obstaculizan el disfrute y ejercicio equitativo de los derechos ciudadanos por parte de hombres y mujeres (Género, s/d). Estas diferencias son por las oportunidades, acceso, control y uso de los recursos que permiten garantizar el bienestar y desarrollo humano.

Para la medición de estas brechas el Programa de las Naciones Unidas para el Desarrollo creó un índice que tiene como finalidad la medir la desigualdad entre hombres y mujeres. Se denomina Índice de Desigualdad de Género (IDG) y se interpreta como la pérdida potencial de desarrollo humano en tres dimensiones: mercado laboral, empoderamiento y salud reproductiva, a través de cinco indicadores: participación en la fuerza laboral, nivel de instrucción, escaños en el parlamento, fecundidad adolescente y mortalidad materna. Se expresa como porcentaje y entre más cercano esté al 1 es indicativo de igualdad entre hombres y mujeres (PNUD, 2014a). 


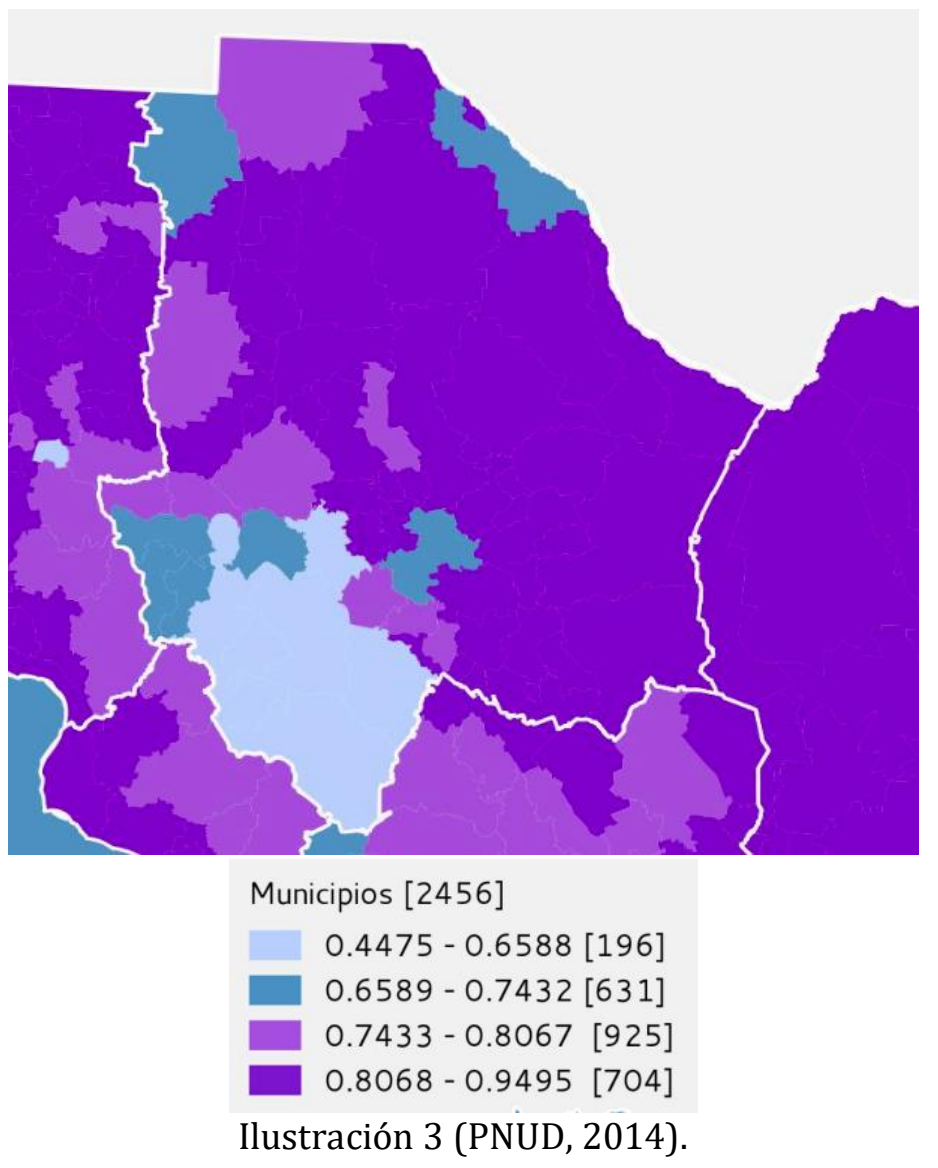

En la ilustración 3 se puede apreciar que la región en la cual se realizó la investigación se cuenta con un nivel bajo de IDG. Batopilas y Balleza municipios chihuahuenses se localizan entre los 10 municipios de México con mayor desigualdad en el aspecto de género, es de esperarse entonces que se van a encontrar brechas de género importante en las respuestas ofrecidas por chicos y chicas de la región. Es interesante ampliar la mirada de este índice en aspectos puntuales de la calidad de vida de los y las estudiantes de educación secundaria de la región serrana para establecer las brechas de género de la información generada por la investigación, objetivo de este trabajo.

Para realizar la medición de las variables integrantes del estudio de calidad de vida se realizó un cuestionario de 96 ítem dividido en cuatro categorías que definen la calidad de vida: salud física, mental, contextos educativo y familiar, así como de las competencias para la vida. Los cuestionamientos se realizaron a través de varios ítems de un cuestionario, se exploraron las percepciones del estudiantado a través de las respuestas "nunca", "casi nunca", "a veces", "casi siempre" y "siempre" a preguntas cerradas planteadas.

Se aplicó a una muestra probabilística representativa de los estudiantes de educación secundaria de la región serrana. El universo estuvo integrada por 3800 estudiantes localizados 19 escuelas en las comunidades de: Sisogichi, Creel, San Juanito, Norogachi, Vicente Guerrero, Guachochi, Tonachi, Samachique, Nonoava y Carichi del Estado de Chihuahua. Sin embargo, por condicionantes de la violencia que se vivía en el municipio de Guachochi, se vio la necesidad de suplir estos estudiantes por alumnado de los municipios de Juárez y Chihuahua. De ellos 
se seleccionó una muestra representativa aleatoria compuesta por 400 estudiantes.

El análisis estadístico llevado a cabo para obtener los resultados fueron principalmente estadísticos descriptivos:

- $\quad$ Aplicación de medidas de tendencia central como las Medias las agrupaciones de los datos en cada variable.

- Medidas de dispersión (varianza y desviación estándar), con la finalidad de determinar la variación de los datos en y entre las variables.

\section{El comportamiento de la calidad de vida en jóvenes chihuahuenses a partir del género}

Las principales brechas de género en el estudio fueron encontradas en el aspecto de la Salud y se describen a continuación. Algunas de las preguntas realizadas a través del instrumento no reportaron diferencia, estas se omiten debido a que el trabajo alude a "brechas de género". En el eje de las "y" se expresa el promedio de respuestas ofrecidas por los y las estudiantes. El número cinco da como respuesta "siempre" y el número uno la respuesta que ofrece el estudiantado es "nunca", entre este rango se encuentran los términos medios de la frecuencia a reportar: casi siempre, casi nunca y a veces.

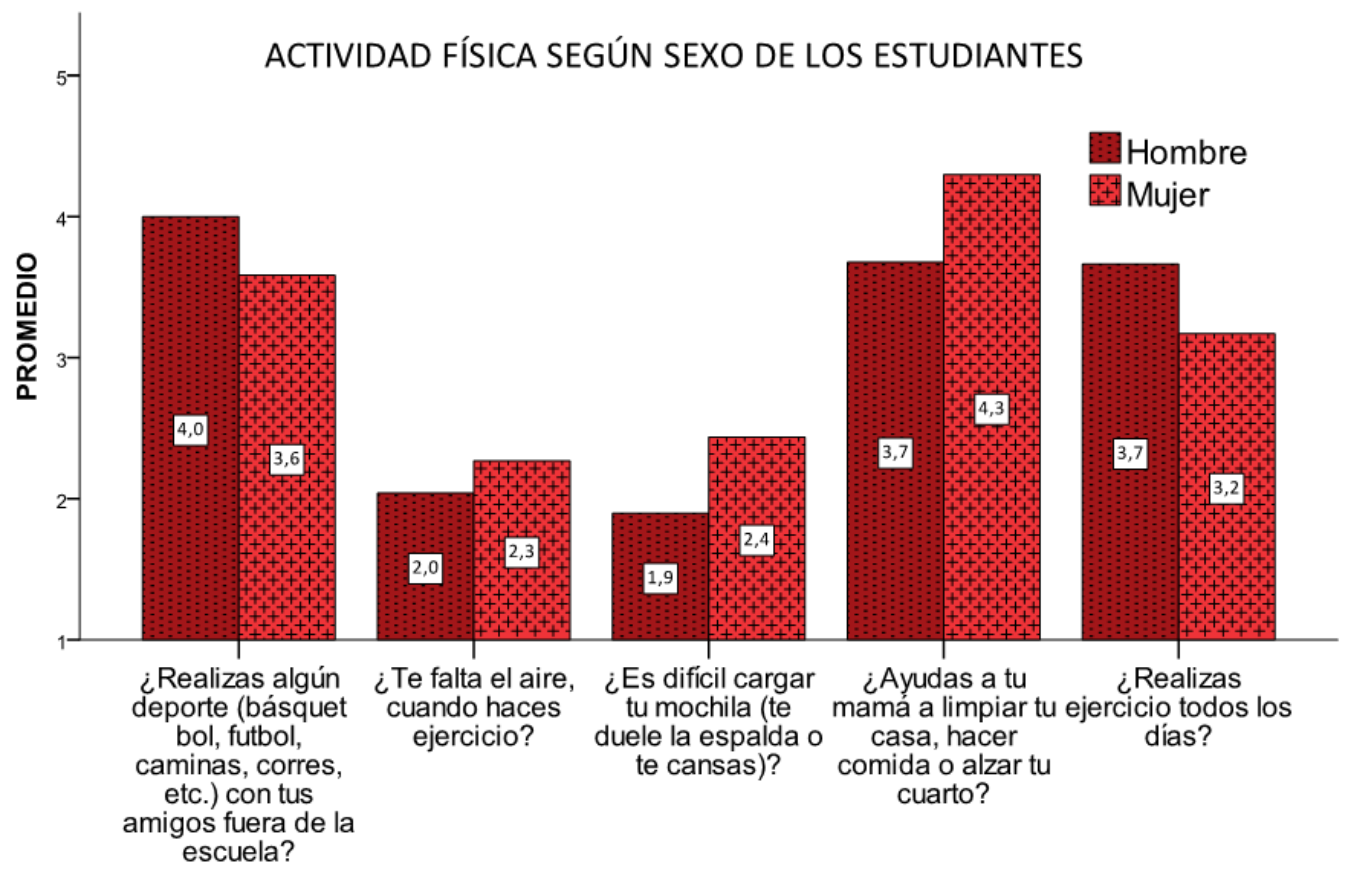

Gráfica 2.

En la gráfica 2 se puede apreciar la presencia de brechas en torno a la activación física del estudiantado: mientras los hombres en promedio reportan más cercana la respuesta a "siempre", las mujeres tienen .4 menos. Esto es indicativo del estereotipo que socialmente se establece en torno a que el deporte es "cosa de hombres". Tal vez esto tenga como consecuencia que las mujeres reporten mayor frecuencia en las preguntas ite falta el aire cuando haces ejercicio? y ¿es difícil cargar tu mochila? La división sexual del trabajo impera entre el estudiantado puesto que la brecha entre los hombres y las mujeres en la realización del trabajo doméstico es de .6. 


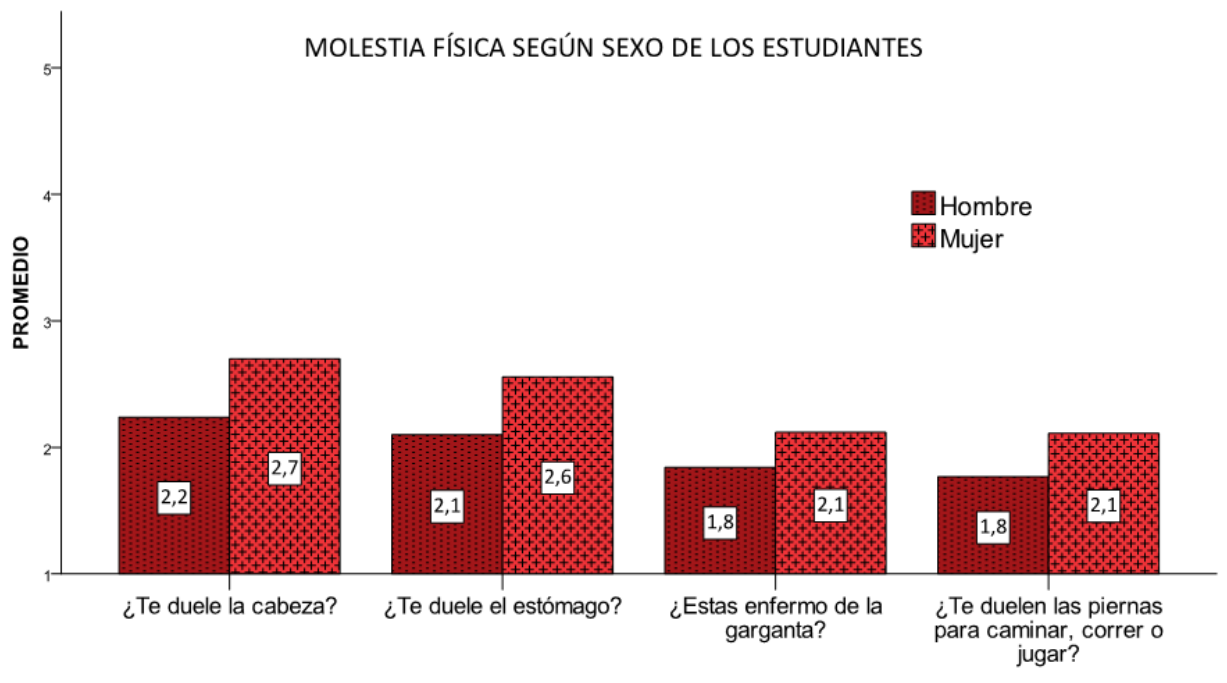

Gráfica 3.

Para una persona que no tiene la mirada de género la gráfica 3 pudiese expresar que las estudiantes de secundaria de la región serrana se enferman más, sin embargo, lo que se puede leer es cómo para las mujeres es mucho más fácil expresar algún tipo de enfermedad o molestia. La masculinidad tradicional impide que el hombre se asuma con debilidad y las molestias físicas son muestra de "debilidad", entonces se es "más hombre" si "no se queja" o "se aguanta" las molestias. En todas las preguntas los hombres asumen menor incidencia de dolores o enfermedades que las mujeres.
Nuevamente los estereotipos de género privan en la información que ofrece la gráfica 4, puesto que son las mujeres quienes gozan de la posibilidad social de ser limpias, ordenadas y de expresarlo abiertamente. Preocupa no solamente la diferencia en las respuestas de hombres $y$ mujeres que expresan recibir alimentos antes de ir a la escuela, en la que las mujeres son desfavorecidas en un .3, sino que la respuesta no sea unánime en ambos en torno a recibir siempre comida previa a la escuela.

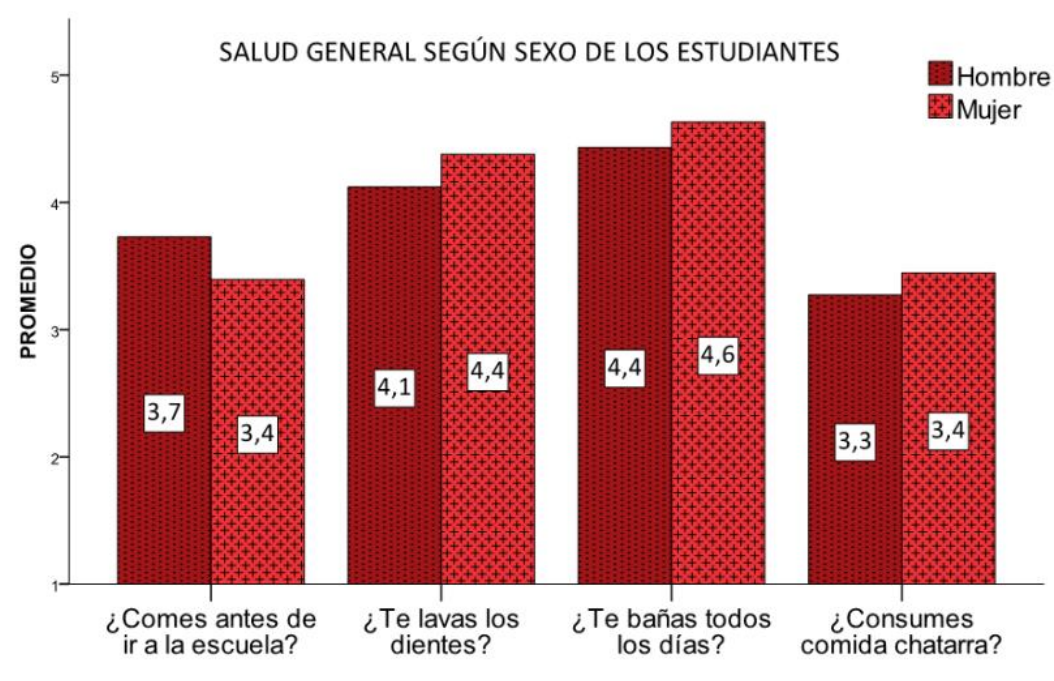

Gráfica 4. 


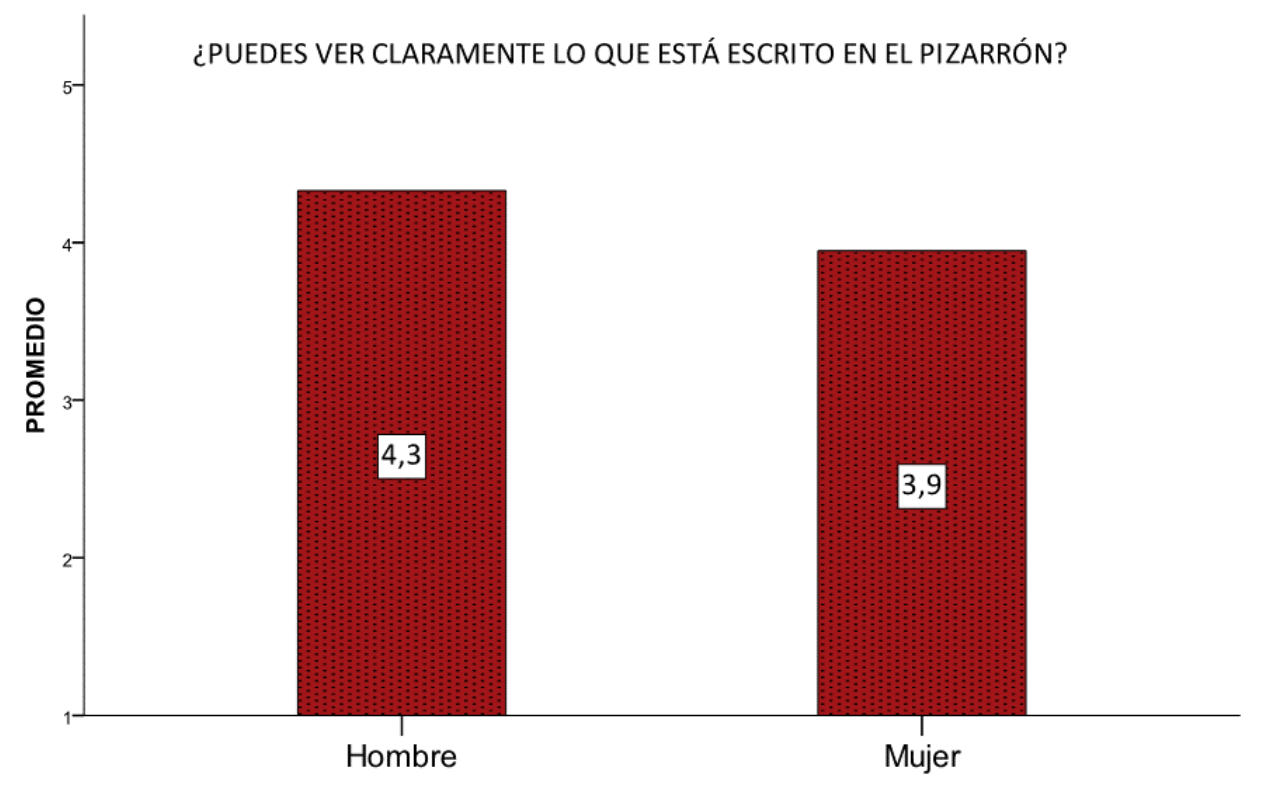

Gráfica 5.

La gráfica 5 comunica una brecha en las respuestas que brindan chicas y chicas en torno a la agudeza visual, .4 de diferencia en respuestas sobre la visión con claridad de lo que se escribe en el pizarrón. Nuevamente es necesario profundizar en la posibilidad de naturalizar las diferencias y decir que los hombres ven mejor que las mujeres; es posible pensar que ellos expresan lo que la sociedad quiere que digan al ser más fuertes, mejor dotados, más perfectos.
La cultura de género se hace también presente en la gráfica 6 al informar las mujeres su tendencia al mal humor $\mathrm{y}$ al desgano por hacer las cosas. Este fenómeno no se relaciona con la cuestión hormonal como ligeramente se juzga, sino a situaciones de inferioridad y discriminación que viven las mujeres (Lazarevich, Delgadillo-Gutiérrez, Mora-Carrasco, \& Martínez-González, 20132014). 


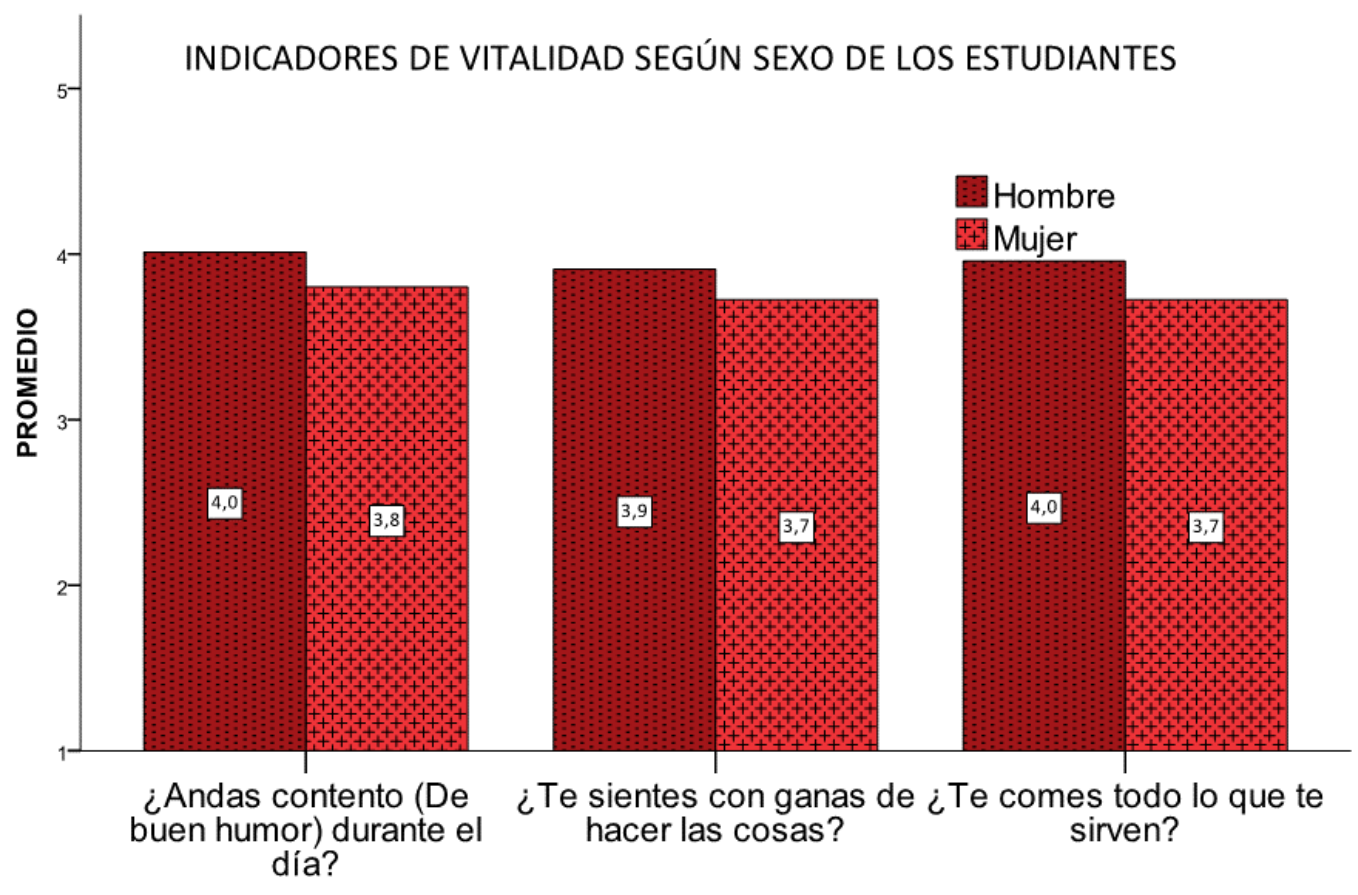

Gráfica 6.

La misma tendencia se observa en la gráfica 7 pues las mujeres expresan mayor temor, inseguridad, enojo y miedo que los hombres. Se reitera: no es que las mujeres presenten mayor incidencia de estos fenómenos, es que las mujeres "tienen permiso de expresarlas más" (Lazarevich et al., 2013-2014).

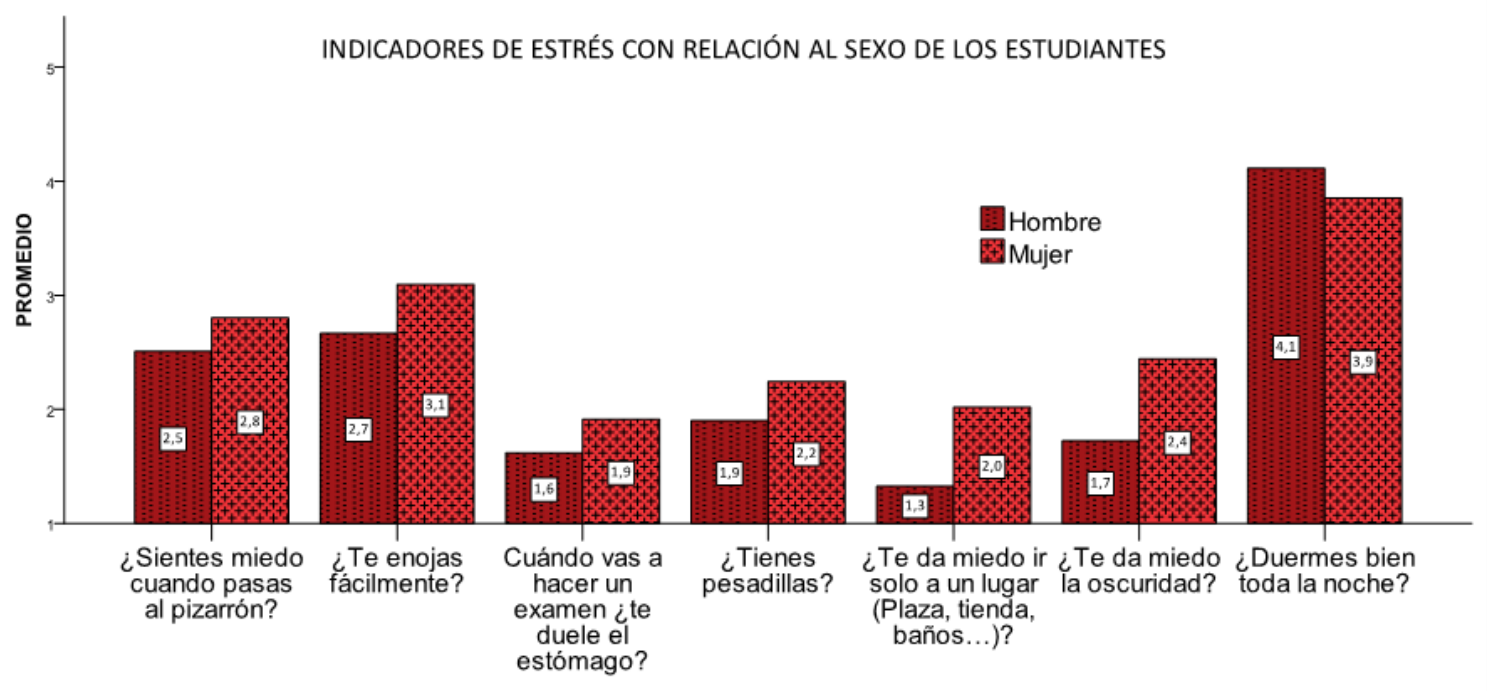

Gráfica 7.

Nuevamente los hombres reportan menor miedo e inseguridad que las mujeres. Esto se observa en la gráfica 8 , en la que preocupa la brecha de un punto porcentual expresada por las mujeres en la pregunta que alude al tratamiento con otras personas. La brecha en síntomas de ansiedad, como lo es "comerse las uñas", también es preocupante con 0.6 de diferencia entre hombres y mujeres. 


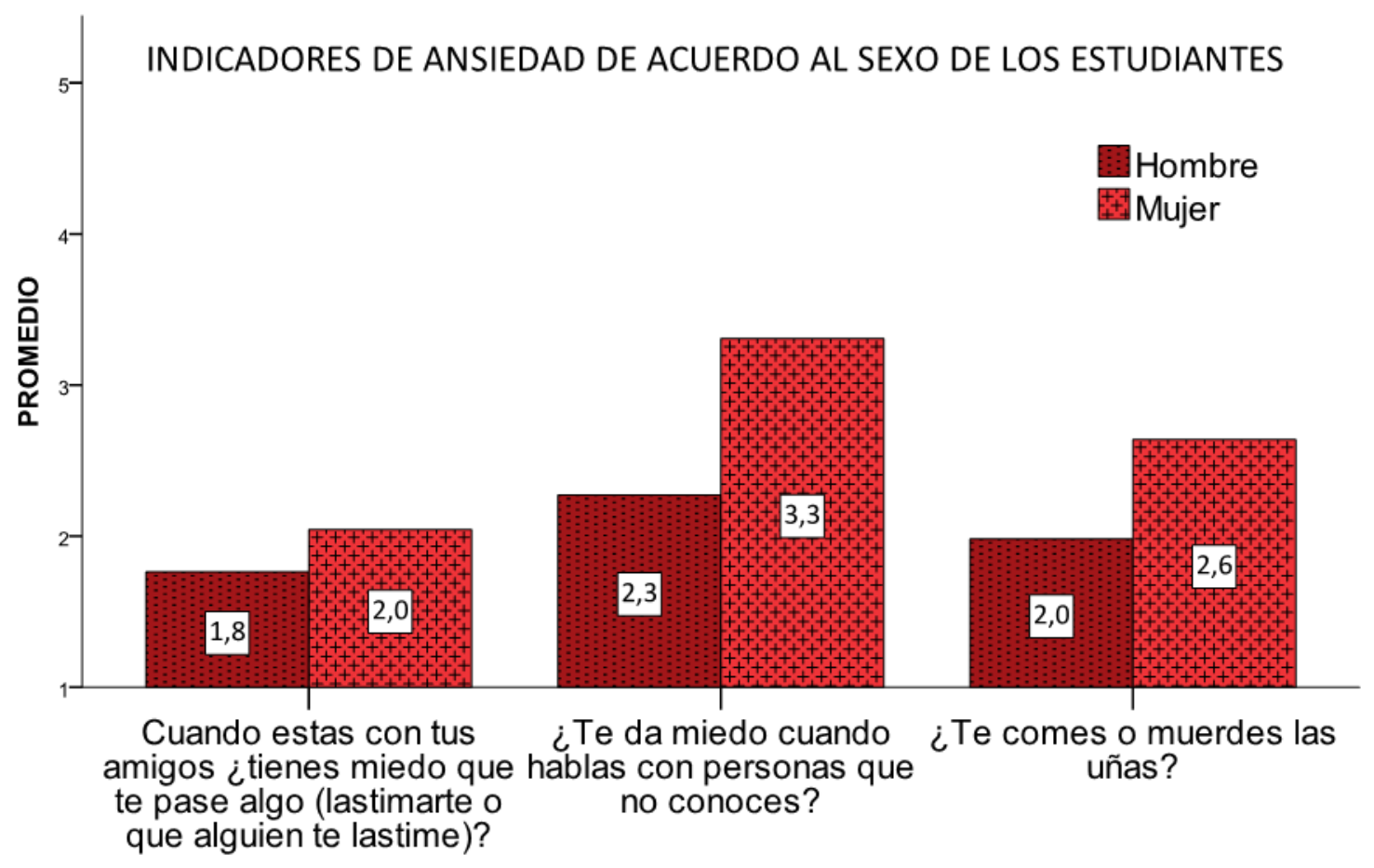

Gráfica 8.

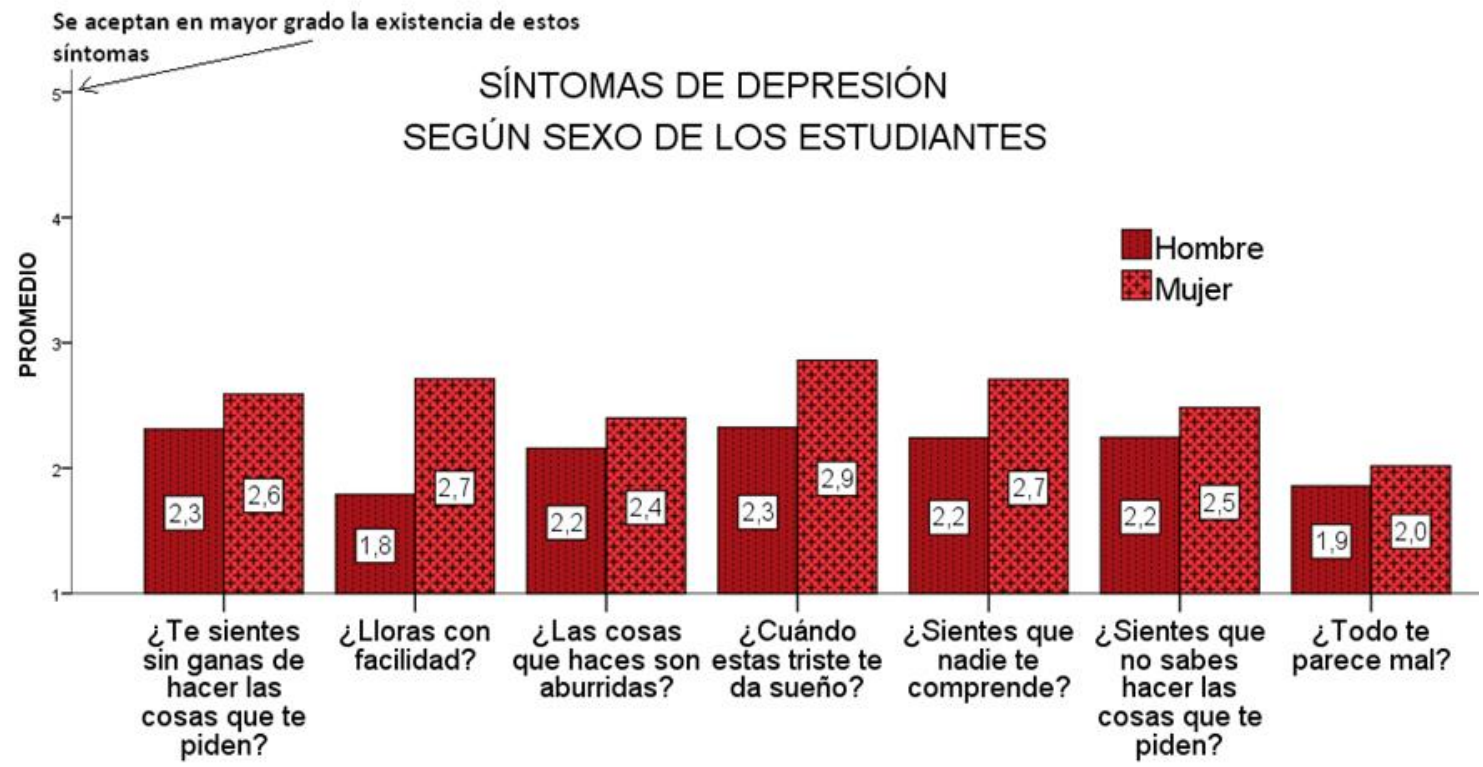

Gráfica 9.

La gráfica 9 nuevamente evidencia el fenómeno de la masculinidad tradicional en los que no está permitido expresar a los hombres la expresión de los sentimientos. La brecha más profunda es en la pregunta ¿Lloras con facilidad? en la que las mujeres expresan mayor frecuencia en mostrar este sentimiento, la brecha se presenta en un 0.9. En todos los casos la brecha entre hombres y mujeres es la mayor expresión de malestar, de aburrimiento, tristeza, incomprensión e ignorancia por parte de las mujeres. 


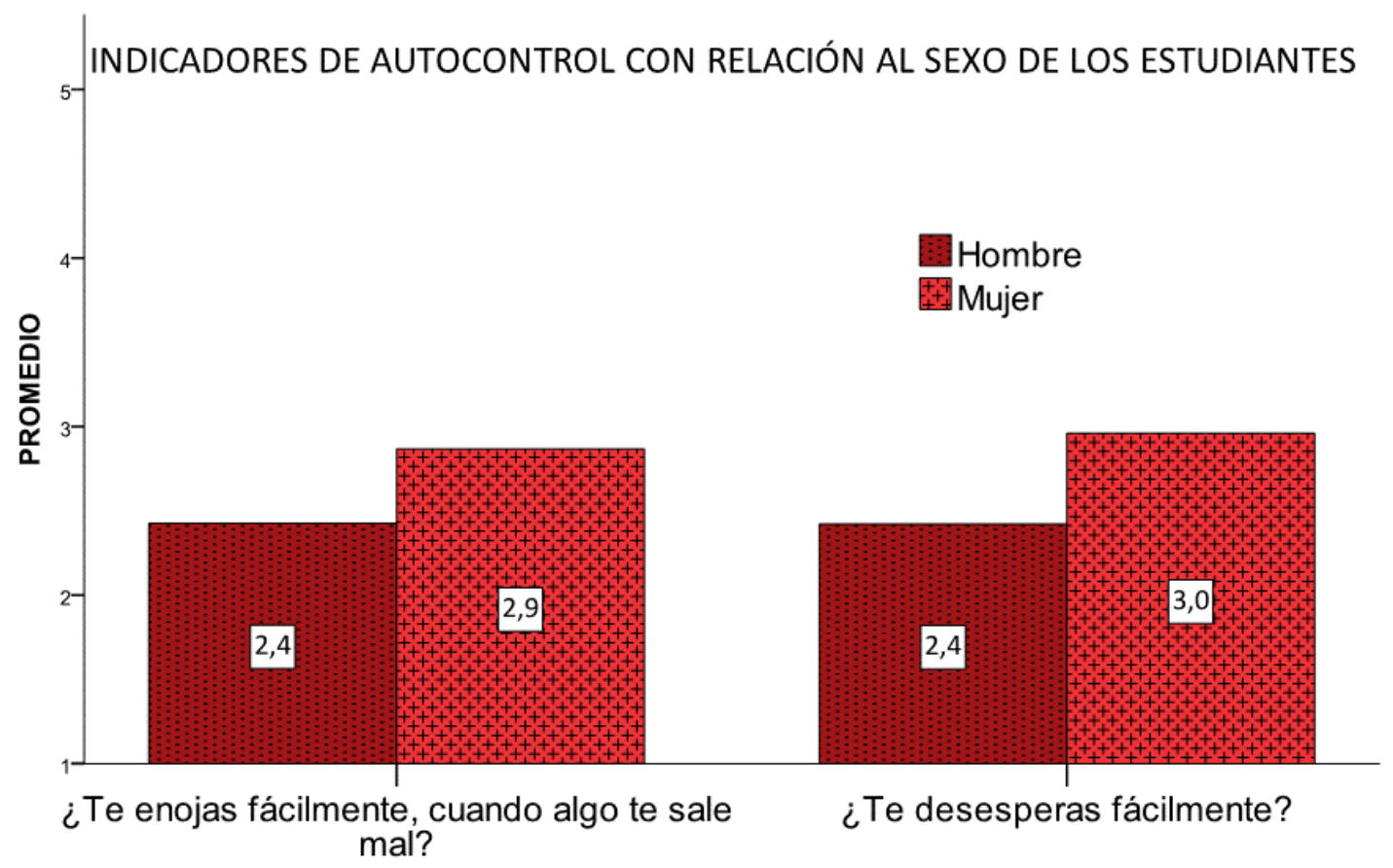

Gráfica 10.

Aunque la violencia y el enojo socialmente está "encarnado" más en el cuerpo de los hombres, no es correcto para la sociedad tradicional "darse por vencido". En el caso de la gráfica 10 se muestra claramente cómo para las mujeres es más fácil informar que se enojan cuando algo sale mal y que se desesperan fácilmente. Como la masculinidad tradicional asume que los hombres deben ser más racionales y objetivos, controlan estos sentimientos o los expresan con mayor dificultad esto origina en los hombres problemáticas severas y violencia de salud (Bonino, 2004; Sabo, s/d).
Por último, en la gráfica 11 al cuestionársele a los y las jóvenes sobre la salud sexual y reproductiva las respuestas ponen en desventaja a los hombres pues reportan menor información o apertura a expresar información sobre esta temática. Las mujeres refieren contar con mayor información, con mayor posibilidad de apertura a trabajar la temática. Sin embargo, también se puede observar cómo la escuela es quien posibilita mayormente la comunicación en torno a información sexual y la familia se queda al margen de ello. Esto evidencia la dificultad en el tratamiento de este tema considerado tabú. 


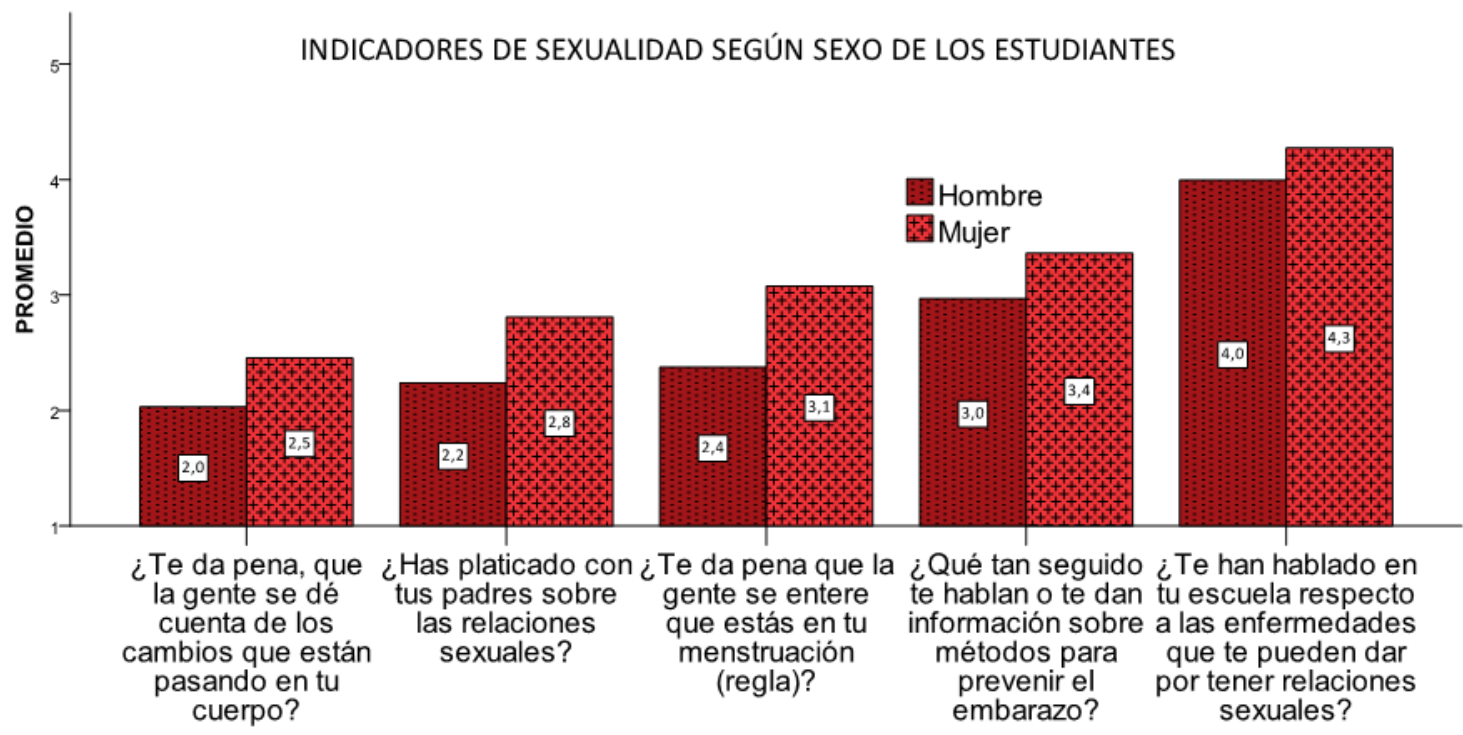

Gráfica 11.

\section{A manera de conclusión}

Durante la exposición de los resultados de la investigación se han establecido resultados encontrados en la búsqueda de las percepciones que tienen que ver con la calidad de vida y la educación de chicos y chicas chihuahuenses de educación secundaria ubicadas en la región serrana. Se encontró desigualdad en los indicadores de hombres y mujer que producen brechas de género. $\mathrm{Si}$ bien las diferencias se dan como "fenómenos naturales" o las explicaciones se alojan en situaciones biológicas "propias" del hombre y la mujer, se fundamentan en construcciones culturales establecidas en un marco histórico social.

Las brechas más pronunciadas se dan en los aspectos emocionales en las que hay hasta un punto porcentual de diferencia en los sentimientos de hombres y mujeres. Las mujeres asumen inseguridades, miedos, llantos, enojos, depresión, ansiedad de manera más frecuente que los hombres. Estudios de masculinidad fundamentados en la Perspectiva de Género (Bonino, 2004; Lazarevich et al., 2013-2014; Milicua, 2009; Sabo, s/d) brindan explicaciones en torno a lo que la sociedad considera "no deseable" en los rasgos de la masculinidad tradicional la expresión de sentimientos y la influencia en la salud. Desde la tradición, los hombres deben ser fuertes, valientes, seguros de sí mismos por lo que es criticado por la sociedad cuando muestran debilidad, inseguridad y todo lo relacionado con los sentimientos. Esto explica las no asistencias al médico, la reticencia a realizarse análisis clínicos y el fenómeno que aquí se encontró: los jóvenes expresan en menor medida que las mujeres, el sentir hacia este tipo de aspectos.

En cambio, existen brechas de desigualdad que afectan a los hombres. Una de ellas es en torno a la información que sobre sexualidad se les brinda. Las mujeres expresan mayor frecuencia que reciben información sobre estos temas con su familia y en la escuela, en detrimento de lo expresado por los hombres que asumen menor frecuencia este fenómeno. En los hábitos de higiene también se aprecian ligeras diferencias que ponen en desventaja a la masculinidad.

Todas las brechas de género pueden ser preocupantes, sin embargo, una de éstas que destaca más se refiere a la alimentación y la ejercitación, porque las mujeres refieren 
que se alimentan antes de asistir a la escuela con menor frecuencia que los hombres. También sobre la ejercitación del cuerpo las mujeres refieren desventaja, la explicación la brinda la perspectiva de género que denuncia cómo el deporte o la activación física se asume como deseable más al género masculino que al femenino.

Las explicaciones culturales se dan desde aspectos estructurales. Recuérdese que en la zona el Î́ndice de Desarrollo de Género es de los más desiguales que existen en la entidad. Quedan muchos pendientes en el trabajo que sobre nuevas rutas de masculinidad y masculinidad tiene que realizar la sociedad. La educación debe ser una posible constructora.

\section{Referencias}

Aroila, R. (2003). Calidad de vida: una definición integradora. Revista Latinoamericana de Psicología, 35 - No. $2,161-164$.

Bonino, L. (2004). Masculinidad, salud y sistema sanitario -el caso de la violencia masculina-. In R. Jarabo \& B. P (Eds.), Seminarios sobre Mainstreaming de género en las políticas de salud en Europa. Madrid: Díaz de Santos.

Género, U. d. I. d. (s/d). Rincón autodidacta. Recuperado de: https://www.juntadeandalucia.es/inst itutodelamujer/ugen/modulos/Indica dores/bgenero.html

INEE. (2013). Panorama Educativo de México. Indicadores del Sistema Educativo Nacional. Educación Básica y Media Superior. México: INEE.

INEGI. (2004). La población hablante de lengua indígena de Chihuahua. Aguascalientes México: INEGI.

Jarque, C. (2001). Los grandes retos de la educación secundaria en América
Latina y el Caribe. Trabajo presentado en el Seminario "Alternativas de reforma de la educación secundaria", Santiago de Chile. http://www.oei.es/reformaseducativa s/retos_educacion_secundaria_AL_cari be_jarque.pdf

Lamas, M. (1995). Usos, dificultades y posibilidades de la categoría género. La Ventana. Estudios de Género, I.

Lamas, M. (1996). La antropología feminista y la categoría género. En PUEG (Ed.), El género: la construcción cultural de la diferencia sexual. México: PUEG/Editorial Porrúa.

Lazarevich, I., Delgadillo-Gutiérrez, H., MoraCarrasco, F., \& Martínez-González, Á. (2013-2014). Depresión, autoestima y características de personalidad asociadas al género en estudiantes rural de México. Alternativas en Psicología, Año XVII.

López, F., \& Angulo, R. (2006). La Reforma de la Educación Secundaria en México. Revista Mexicana de Investigación Educativa, 11(31), 1427-1450.

Milicua, I. A. (2009). Salud y Masculinidad. S/D: Gizonduz/EMAKUNDE.

Oswald-Spring, U., Serrano-Oswald, S., Estrada-Álvarez, A., Flores-Palacios, F., Ríos-Everardo, M., Günter, H., EstradaVillanueva, A. (2004). Vulnerabilidad social $y$ género entre migrantes ambientales. México: UNAM.

PNUD. (2014a). Indicadores de Desarrollo Humano y Género en México: nueva metodología. México: PNUD.

PNUD. (2014b). Índice de Desarrollo Humano Municipal en México: nueva metodología (O. d. I. e. D. H. d. P. d. l. N. U. p. e. D. e. México Ed.). México: Programa de las Naciones Unidas para el Desarrollo.

PNUD. (S/D). Human development Reports. El índice de Desarrollo Humano (IDH). 
Recuperado

http://hdr.undp.org/es/content/el\%C3\%ADndice-de-desarrollohumano-idh

Román, M. (2009). Abandono y deserción escolar: Duras evidencias de la incapacidad de retención de los sistemas y de su porfiada inequidad. Revista Iberoamericana sobre Calidad, Eficacia y Cambio en Educación, 7(4), 4-9.

Sabo, D. (s/d). El estudio de masculinidad, género y salud. Comprender la salud de los hombres.

Schmelkes, S. (2012). Los grandes problemas de la educación básica en México. Recuperado de: http://www.inidedelauia.org/2012/0 $1 /$ los-grandes-problemas-de-laeducacion.html
Tedesco, J. C., \& López, N. (2002). Desafíos a la educación secundaria en América Latina. Revista de la CEPAL.

Zorrilla Fierro, M. (2004). La educación secundaria en México: al filo de su reforma. REICE: Revista Electrónica Iberoamericana sobre Calidad, Eficacia y Cambio en Educación.

\section{Agradecimientos}

Este estudio fue realizado con la colaboración de la Facultad de Medicina de la UACH y los Servicios Educativos del Estado de Chihuahua. Participó también el Departamento de Educación Secundaria y el Departamento de Educación Telesecundaria de la Dirección de Educación Básica de la Secretaría de Educación y Cultura. 
RECIE. Revista Electrónica Científica de Investigación Educativa Vol. 3, núm. 2, enero-diciembre 2017, pp. 919-933. 